\title{
Alguns aspectos da biologia de Triatoma pseudomaculata Corrêa \& Espínola, 1964, em condições de laboratório (Hemiptera:Reduviidae:Triatominae)
}

\author{
Teresa Cristina M Gonçalves ${ }^{+}$, Vanda Cunha, Edson de Oliveira, José Jurberg
}

\author{
Laboratório Nacional e Internacional de Referência em Taxonomia de Triatomíneos, Departamento de \\ Entomologia, Instituto Oswaldo Cruz, Av. Brasil 4365, 21045-900 Rio de Janeiro, RJ, Brasil
}

\begin{abstract}
Biology of Triatoma pseudomaculata Corrêa \& Espínola, 1964, under Laboratory Conditions (Hemiptera:Reduviidae:Triatominae) - Observations were made on the evolutive cycle of Triatoma pseudomaculata, held under laboratory conditions, fed weekly on bird (pigeon). Of 60 eggs obtained, only 34 nymphs reached the adult stage in a period of $\bar{X}(S)=398 \pm 76$ days. The following parameters were observed: the time immature stages took to develop from egg to adult emergence; the occurrence of the first meal; the time-lapse between the presenting of the blood-meal and the begining of feeding; time of feeding; amount of blood ingested; variation of weight $24 \mathrm{hr}$ after the blood-meal and until the next blood-meal; and the defecation pattern. The experiment was carried out for 20 months, held in $B O D$ incubator with the average of temperature and humidity of $28 \pm 1^{\circ} \mathrm{C}$ and $80 \pm 5 \% R U$, respectively.
\end{abstract}

Key words: Triatoma pseudomaculata - biology - laboratory conditions

Lucena (1959) refere-se a Triatoma maculata (Erichson, 1848), posteriormente Triatoma pseudomaculata Corrêa \& Espínola, 1964, como uma espécie de importância regional, encontrada em pombais, galinheiros e cercas, sempre no peridomicílio e nunca em abrigos silvestres, e como característica da fauna nordestina, distribuída geograficamente pelos Estados de Pernambuco, Paraíba, metade do Ceará, sertão de Alagoas, Bahia, Minas Gerais, Piauí e Goiás. Silveira et al. (1984) relatam a ampla distribuição geográfica dessa espécie, comentam sua baixa freqüência nas habitações, embora colonize algumas casas, e informam que, possivelmente, é o vetor do Trypanosoma cruzi em algumas áreas da Paraíba. Dados da Fundação Nacional de Saúde, não publicados, mostram que entre as sete espécies presentes no Estado de Pernambuco, infestando o domicílio humano, ela ocupa o segundo lugar, porém com baixo índice de infecção (dos 1.709 espécimes capturados, $0,5 \%$ estava positivo). Este resultado pode estar de acordo com as observações de Perlowagora-Szumlewicz e Müller (1982) e Perlowagora-Szumlewicz et al. (1988) pois,

Financiado pelo PAPES, FAPERJ, CNPq, Convênio 027/93 FNS/BIRD/FIOCRUZ

+Autor correspondente. Fax: +55-21-590.9339.E-mail: Gonçalves@DCC001.CICT. FIOCRUZ.BR

Recebido em 11 de junho de 1996

Aceito em 25 de novembro de 1996 segundo esses autores, a domiciliação altera a relação vetor-parasita reduzindo o índice de infecção e a carga parasitária.

Dias (1991) comenta sobre a dificuldade da erradicação dessa espécie, uma vez que é nativa, ubiqüista e ocorre em grandes quantidades no meio extradomiciliar, especialmente em regiões pobres e isoladas da área endêmica.

Forattini et al. (1981) classificaram-na como de moderada tendência à domiciliação mas com elevada ornitofilia, embora o estudo da preferência alimentar tenha-lhe atribuído um ecletismo alimentar.

Apesar de incluída entre as cinco espécies mais importantes pela transmissão da doença de Chagas no Brasil, foram poucos os estudos feitos a respeito de sua biologia (Heitzmann-Fontenelle 1972, Perlowagora-Szumlewicz 1976).

Neste trabalho, procurou-se conhecer a biologia dessa espécie utilizando como fonte alimentar a ave, tendo em vista, não só a ornitofilia já observada por Forattini et al. (1981), como também procurouse evitar as modificações de parâmetros físiológicos ocasionadas por alimentação artificial (De Isola et al. 1980). Espera-se, com esses resultados, obter informações que contribuam para melhorar a compreensão da epidemiologia da doença de Chagas nas áreas de ocorrência de T. pseudomaculata, e que facilitem o planejamento de ações de controle, uma vez que a sua presença no peridomicílio possibilitará constantes invasões dos domicílios. 


\section{MATERIAIS E MÉTODOS}

$\mathrm{O}$ experimento iniciou-se com 60 ovos, que foram acondicionados em frascos, por postura; conforme ocorriam as eclosões, as ninfas eram individualizadas em frascos de Borrel, devidamente identificados, contendo papel de filtro no fundo e dobrado em sanfona, para retirar o excesso de umidade proveniente das fezes e para aumentar a área livre.

A alimentação foi oferecida, semanalmente, com sangue de pombo (Columba livia). Para isso, depenava-se uma pequena área do flanco suficiente para a acomodação do frasco de Borrel. Para as ninfas de $1^{\circ}$ estádio o oferecimento foi diário, a fim de se observar a ocorrência do primeiro repasto.

Foram avaliados os seguintes parâmetros: período de incubação; duração de cada estádio até a fase adulta; realização do primeiro repasto; intervalo de tempo entre o oferecimento e o início do repasto; duração do repasto; quantidade de sangue ingerida; perda de peso até $24 \mathrm{~h}$ após o repasto e até o próximo, em relação ao peso inicial; número de defecações por estádio, em um período de até 10 min. após a alimentação.

$\mathrm{O}$ experimento teve a duração de 20 meses e foi realizado em estufa BOD a $28 \pm 1^{\circ} \mathrm{C}, 80 \pm 5 \%$ UR e fotoperíodo de $12 \mathrm{~h}$. A pesagem foi realizada em balança de precisão.

O material trabalhado pertencia a uma colônia procedente de Morada Nova (CE) mantida no insetário do Laboratório Nacional e Internacional de Referência em Taxonomia de Triatomíneos do Departamento de Entomologia do Instituto Oswaldo Cruz.

\section{RESULTADOS}

Dos 60 ovos obtidos, apenas 34 espécimes (16 fêmeas e 18 machos) atingiram a fase adulta. Os demais (doze ninfas de $1^{\circ}$ estádio, quatro de $2^{\circ}$ e $4^{\circ}$ estádios e três de $3^{\circ}$ e $5^{\circ}$ e estádios) morreram, na sua maioria, por não conseguirem se alimentar, apesar de várias investidas sem sucesso, ou por motivo inexplicável (três casos).

A duração do ciclo do 10 estádio a adulto foi de $\bar{X}(S)=398 \pm 76$ dias, com o mínimo e máximo de 269 e 532 dias, respectivamente. Do $1^{\circ}$ estádio até a ecdise imaginal das fêmeas, o ciclo foi de $\bar{X}(S)=412 \pm 77$ dias e o dos machos de $\bar{X}(S)=$ $385 \pm 74$ dias, o que demonstra haver uma geração por ano.

$\mathrm{Na}$ Tabela I, onde são apresentados os resultados referentes à duração de cada fase do desenvolvimento, observa-se que o tempo requerido foi crescente até o $4^{\circ}$ estádio, decaindo do $5 \underline{0}$ até a fase de adultos, com as fêmeas seguidas dos machos. A observação dos adultos foi realizada até a constatação da morte.
As ninfas de $1 \underline{o}$ estádio realizaram o primeiro repasto entre o $1^{\underline{0}}$ e $17 \underline{0}$ dias, com maiores incidências no $2^{\mathrm{o}}(15 \%), 4^{\circ}, 11^{\circ} \mathrm{o}$ e $12^{\mathrm{o}}$ dias $(12 \%)$. As alimentações subseqüentes ocorreram no $70 \mathrm{o}$ e $8^{\circ}$ dias $(8 \%) ; 3^{\circ}, 5^{\circ}$ e $9^{\circ}$ o dias $(6 \%)$ e $1^{\circ}, 6^{\circ}, 10^{\circ}$, $13^{\circ}$ e 17 - dias (3\%).

O número de repastos por espécime, durante todo o ciclo de vida, distribuiu-se homogeneamente entre um mínimo de 10 (uma fêmea) e máximo de 32 (duas fêmeas). As fêmeas efetuaram, em média, maior número de repastos, seguidas pelos machos e pelas

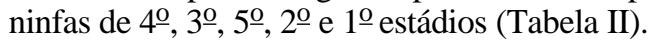

$\mathrm{O}$ intervalo de tempo entre o oferecimento do repasto e o início da alimentação aumentou, gradativamente, de acordo com a evolução do estádio ninfal, decaindo na fase adulta (Tabela III).

A duração do repasto (Tabela IV) foi crescente do $1^{\circ} \mathrm{o}$ ao $4 \stackrel{0}{ }$ estádios, seguidos por machos, fêmeas e 5ํㅡㄹ. estádio.

A quantidade de sangue ingerida (Tabela V) foi crescente até o 3 o estádio, seguido por machos, $4^{\mathrm{o}}$ estádio, fêmeas e $5^{\circ} \mathrm{o}$ estádio; não foi observada diferença desse parâmetro entre as ninfas que evoluíram para qualquer dos dois sexos.

Os resultados da Tabela VI demonstram que, na fase ninfal, a perda de peso foi sensivelmente

\section{TABELA I}

Duração do ciclo de vida de Triatoma pseudomaculata (dias)

\begin{tabular}{lrrrr}
\hline Fase de & \multicolumn{3}{c}{ Amplitude } & Desvio \\
\cline { 2 - 3 } desenvol. & Mínima & Máxima & Média & padrão \\
\hline Incubação & 15 & 21 & 18 & 1 \\
1o estádio & 19 & 85 & 44 & 18 \\
20 estádio & 27 & 102 & 49 & 22 \\
30 estádio & 36 & 101 & 69 & 22 \\
4o estádio & 45 & 134 & 87 & 28 \\
50 estádio & 43 & 137 & 82 & 27 \\
Fêmeas & 19 & 125 & 71 & 27 \\
Machos & 32 & 109 & 67 & 21 \\
Fêmeas e machos & 19 & 125 & 67 & 24 \\
\hline
\end{tabular}

TABELA II

Número de repastos realizados em diferentes fases do desenvolvimento de Triatoma pseudomaculata

\begin{tabular}{lrrrr}
\hline Fase de & \multicolumn{3}{c}{ Amplitude } & Desvio \\
\cline { 2 - 3 } desenvol. & Mínima & Máxima & Média & padrão \\
\hline 1o estádio & 2 & 4 & 2,4 & 0,8 \\
20 estádio & 1 & 7 & 2,6 & 1,1 \\
3o estádio & 2 & 7 & 3,2 & 1,1 \\
4o estádio & 2 & 7 & 3,5 & 1,1 \\
5o estádio & 2 & 7 & 3,0 & 1,3 \\
Fêmeas & 0 & 13 & 5,1 & 3,1 \\
Machos & 1 & 12 & 3,8 & 3,1 \\
\hline
\end{tabular}




\section{TABELA III}

Intervalo de tempo entre o oferecimento do repasto e o ato de alimentação de Triatoma pseudomaculata (min./seg.)

\begin{tabular}{lcccr}
\hline Fase de & \multicolumn{3}{c}{ Amplitude } & Desvio \\
\cline { 2 - 4 } desenvol. & Mínima & Máxima & Média & padrão \\
\hline 1o estádio & $0: 00$ & $15: 05$ & $2: 13$ & $2: 25$ \\
2o estádio & $0: 00$ & $12: 10$ & $2: 17$ & $2: 18$ \\
3o estádio & $0: 00$ & $19: 20$ & $3: 45$ & $3: 32$ \\
4o estádio & $0: 00$ & $18: 35$ & $4: 24$ & $3: 21$ \\
5o estádio & $0: 00$ & $20: 20$ & $5: 55$ & $3: 53$ \\
Fêmeas & $0: 50$ & $12: 00$ & $3: 45$ & $1: 15$ \\
Machos & $0: 00$ & $16: 30$ & $5: 13$ & $2: 27$ \\
\hline
\end{tabular}

TABELA IV

Duração do repasto de Triatoma pseudomaculata (min./seg.)

\begin{tabular}{lccrr}
\hline Fase de & \multicolumn{3}{c}{ Amplitude } & Desvio \\
\cline { 2 - 4 } desenvol. & Mínima & Máxima & Média & padrão \\
\hline 1o estádio & $01: 43$ & $19: 19$ & $09: 00$ & $3: 55$ \\
2o estádio & $02: 22$ & $25: 30$ & $10: 46$ & $5: 14$ \\
3o estádio & $03: 23$ & $39: 00$ & $12: 18$ & $6: 51$ \\
4o estádio & $03: 29$ & $32: 30$ & $13: 58$ & $6: 46$ \\
5o estádio & $06: 15$ & $45: 56$ & $19: 34$ & $8: 13$ \\
Fêmeas & $10: 26$ & $30: 00$ & $18: 21$ & $4: 16$ \\
Machos & $06: 00$ & $41: 37$ & $16: 01$ & $5: 00$ \\
\hline
\end{tabular}

\section{TABELA V}

Quantidade de sangue ingerida (mg) pelo Triatoma pseudomaculata

(peso posterior - peso anterior ao repasto)

\begin{tabular}{lcc}
\hline $\begin{array}{l}\text { Fase de } \\
\text { desenvolvimento }\end{array}$ & Média & Desvio padrão \\
\hline 1o estádio & 2,1 & 1,0 \\
2o estádio & 4,9 & 2,5 \\
3o estádio & 11,9 & 5,9 \\
4o estádio & 28,8 & 13,9 \\
5o estádio & 80,9 & 39,4 \\
Fêmeas & 36,1 & 13,8 \\
Machos & 27,1 & 9,6 \\
\hline
\end{tabular}

maior no período de $24 \mathrm{~h}$ após o repasto; ao contrário da fase adulta, quando a maior perda ocorreu no período entre 24 h após um repasto e o próximo.

O parâmetro defecação foi observado no período de $10 \mathrm{~min}$. após o repasto, estimando-se ser este o tempo necessário para o triatomíneo deixar a fonte alimentar. Desconsideraram-se os resultados referentes à fase adulta, porque o tempo requerido foi sensivelmente maior e nem todos os espécimes puderam ser observados.

Dos 509 repastos realizados do $1 \underline{0}$ ao $5 \underline{0}$ estádios, apenas duas ninfas $(0,4 \%)$ defecaram sobre a fonte alimentar, $19(3,7 \%)$ logo após o repasto, porém fora da fonte e $146(28,6 \%)$ no período estabelecido de $10 \mathrm{~min}$. Os $342(67,2 \%)$ restantes não defecaram naquele período. Observou-se que nas ninfas de $1^{\underline{0}}, 2^{2} \underline{0}, 3^{3} \underline{0}, 4^{\circ}$ e $5^{\underline{o}}$ estádios os índices de defecação foram, respectivamente, $10 \%, 9 \%, 7 \%, 4 \%$ e $2 \%$. Na fase ninfal, no período de $10 \mathrm{~min}$., obtiveram-se os seguintes resultados: 2 e 8 dejeções - 3\%; 9 - 6\%; 7 - 9\%; 3 e 6 - 18\%; 4 - 21\% e 5 - 24\%. A média de defecação dos 34 espécimes neste período de desenvolvimento foi de $\bar{X}(S)=5 \pm 2$. As ninfas que originaram fêmeas e machos apresentaram resultados semelhantes: $\bar{X}=4,94 \pm 1,85$ e $\bar{X}=5,12$ $\pm 1,65$, respectivamente.

\section{DISCUSSÃO}

Os diferentes aspectos do comportamento dos triatomíneos têm sido estudados por diversos autores, especialmente aquelas espécies que participam ativamente na transmissão da doença de Chagas.

Os resultados obtidos neste experimento diferem dos encontrados por Heitzmann-Fontenelle (1972) e Perlowagora-Szumlewicz (1976), possivelmente devido à variação genética entre as populações utilizadas, ao tempo em que a colônia foi mantida em laboratório e às diferentes condições em que foram realizadas as observações.

\section{TABELA VI}

Variação do peso de Triatoma pseudomaculata, 24 h após o repasto e entre o último e o próximo repasto, com as respectivas porcentagens de perda de peso em relação ao peso inicial (mg)

\begin{tabular}{|c|c|c|c|c|c|c|}
\hline \multirow[b]{2}{*}{$\begin{array}{l}\text { Fase de } \\
\text { desenvolvimento }\end{array}$} & \multicolumn{3}{|c|}{$\begin{array}{c}24 \text { h após o repasto } \\
\text { (peso após - peso } 24 \text { h após o repasto) }\end{array}$} & \multicolumn{3}{|c|}{$\begin{array}{r}\text { Entre o último e o próximo repasto } \\
\text { (peso após - peso anterior ao repasto) }\end{array}$} \\
\hline & Média & $\begin{array}{l}\text { Desvio } \\
\text { padrão }\end{array}$ & $\%$ & Média & $\begin{array}{l}\text { Desvio } \\
\text { padrão }\end{array}$ & $\%$ \\
\hline 10 estádio & 0,8 & 0,5 & 38 & 0,0 & 0,0 & 0 \\
\hline 2o estádio & 2,2 & 1,2 & 45 & 2,7 & 1,2 & 55 \\
\hline 30 estádio & 5,8 & 3,0 & 49 & 7,3 & 3,5 & 61 \\
\hline 4ㅇ estádio & 13,1 & 7,0 & 45 & 17,8 & 7,2 & 62 \\
\hline 5o estádio & 41,5 & 22,9 & 51 & 49,2 & 22,5 & 61 \\
\hline Fêmeas & 10,5 & 8,3 & 29 & 50,1 & 40,2 & 139 \\
\hline Machos & 8,4 & 7,3 & 31 & 50,9 & 37,9 & 188 \\
\hline
\end{tabular}


Heitzmann-Fontenelle (1972) estudou o ciclo biológico de T. pseudomaculata utilizando sangue do homem e do coelho (Oryctolagus cuniculus) como fonte alimentar. A alimentação em ave e camundongo foi tentada, mas sem sucesso porque, no primeiro caso, os triatomíneos não se limitavam à área depenada e no segundo caso, a inquietude do camundongo impossibilitava o repasto. No presente trabalho a alimentação foi realizada em ave e sem dificuldade.

Quanto à duração do ciclo de vida das formas jovens, os resultados do presente trabalho situaramse entre aqueles obtidos por Heitzmann-Fontenelle (1972) e Perlowagora-Szumlewicz (1976); esta última autora utilizou o sangue de galinha (Gallus gallus) como fonte alimentar. Na fase adulta, ambas as autoras observaram que os machos vivem mais do que as fêmeas, discordando dos resultados aqui obtidos.

$\mathrm{Na}$ fase ninfal, os espécimes que originaram fêmeas e machos desenvolveram-se no período de $\bar{X}(S)=68,41 \pm 29,71$ e $\bar{X}(S)=64,14 \pm 28,27$ dias, respectivamente. Juarez (1970a) supõe que o rápido desenvolvimento dos machos possa ser atribuído à necessidade de fecundação precoce das fêmeas, visando a manutenção da espécie.

A procura pelo primeiro repasto por ninfas do 1o estádio, segundo Perlowagora-Szumlewicz (1976), ocorreu após o $7^{\circ}$ dia, enquanto que para Heitzmann-Fontenelle (1972) a maioria ocorreu entre 15 e 20 dias, com o mínimo de 9 e máximo de 26 dias. No presente trabalho estes dados diluíram-se entre o $1^{\mathrm{o}}$ e $17^{\mathrm{o}}$ dias, com maior incidência no $2^{\mathrm{O}}$ dia $(15 \%)$ seguido do $4^{\mathrm{O}}, 11^{\mathrm{O}} \mathrm{e}$ $12^{\mathrm{O}}$ dias (12\%), respectivamente.

Segundo vários autores, um único repasto é suficiente para que ocorra a ecdise, exceto no 5o estádio quando há necessidade de mais de um repasto (Brasileiro e Perondini 1974). Discordando destes autores, e de acordo com HeitzmannFontenelle (1972), os resultados aqui apresentados conferem mais de um repasto para cada estádio, o que pode ser justificado pelas diferentes temperaturas e fontes de alimentação (Juarez 1970b) e pela quantidade de sangue ingerida, necessária para que ocorra a ecdise (HeitzmannFontenelle 1976). As fêmeas, para suprirem a menor quantidade de sangue ingerida, procuraram a fonte alimentar em um curto espaço de tempo e realizaram um maior número de repastos.

Do ponto de vista epidemiológico, o tempo do repasto é importante na medida em que possibilita um contato maior entre vetor e hospedeiro, proporcionando condições para que a defecação ocorra ainda sobre o mesmo, assim como aumentando as chances de o triatomíneo adquirir a infecção, especialmente ao se alimentar em um hospedeiro chagásico crônico. Segundo Heitzmann-Fontenelle (1976) esse parâmetro variou no período ninfal entre 15 e $30 \mathrm{~min}$., tempo este maior do que o obtido no presente trabalho. De acordo com a mencionada autora, o tempo requerido pelos adultos foi maior porque para o repasto completo sugavam pequenas quantidades em várias áreas, raramente ficando repletos, ao contrário do observado neste trabalho, cujo tempo foi menor do que o da ninfa de 5으 estádio. Krinsky (1985), estudando o tempo do repasto de outras espécies, verificou que as fêmeas realizam repasto mais longo, porém menor do que o das ninfas de 5o estádio, conforme os resultados aqui apresentados.

Os resultados referentes à quantidade de sangue ingerida foram sensivelmente menores do que os obtidos por Perlowagora-Szumlewicz (1976), possivelmente devido à idade da colônia e/ou à manipulação excessiva dos espécimes (Tolezano et al. 1984b, Costa e Jurberg 1990). Comparando com os resultados obtidos com outras espécies (Zeledón et al. 1970), T. pseudomaculata ingere uma menor quantidade de sangue, demonstrando não ser uma espécie expoliativa, o que não interfere na capacidade de adquirir a infecção (Perlowagora-Szumlewicz e Müller 1982). Não foi observada diferença na quantidade de sangue ingerida entre as ninfas que evoluíram para adulto, machos e fêmeas, o que discorda dos resultados obtidos por Perlowagora-Szumlewicz (1976) e Tolezano et al. (1984a) em relação a T. infestans.

$\mathrm{Na}$ fase ninfal, a perda de peso foi sensível no período de $24 \mathrm{~h}$ após o repasto, concordando com as observações de Lent e Valderrama (1977) e Cirano e Zeledón (1964) para outras espécies.

A freqüência com que ocorre a defecação, durante e após a alimentação, é de interesse epidemiológico e demonstra a capacidade vetorial da espécie que, de acordo com a literatura (Piesman e Sherlock 1983, Zárate et al. 1984, Gonçalves et al. 1988), pode variar entre as espécies. HeitzmannFontenelle (1972) verificou que T. pseudomaculata só defeca sobre o hospedeiro quando atinge a repleção; quando o faz, gira o corpo $80^{\circ}$, eliminando as fezes próximas ao local da picada e aumentando a chance de transmissão do T. cruzi. No presente trabalho este comportamento não foi observado e o índice de defecação decresceu nas fases ninfais, demonstrando que, quanto mais jovem for a fase, maior o número de defecações; conseqüentemente, são parcas as chances de adquirir e transmitir a infecção (Torres 1915). Para T. pseudomaculata, o parâmetro defecação não se mostrou relevante em relação à sua competência vetorial, porque a perda de peso das ninfas ocorreu na maior parte das vezes após os $10 \mathrm{~min}$. de 
observação, porém no período de $24 \mathrm{~h}$ seguintes ao repasto, e entre os adultos após o período de 24 h seguintes ao repasto, confirmando que são remotas as chances de o hospedeiro ficar exposto às fezes infectadas. De acordo com Piesman e Sherlock (1983) existem outros fatores importantes que contribuem para o estabelecimento da capacidade vetorial: a densidade domiciliar, a afinidade com o hospedeiro e o grau de adaptação ao domicílio humano.

A alta temperatura acelera o processo de alimentação (Wood 1951) e de defecação (Maddrell 1964), fatores de extrema importância do ponto de vista epidemiológico. Tendo em vista que $T$. pseudomaculata tem sua ocorrência assinalada em áreas de clima quente, possivelmente, na natureza, o seu comportamento frente a esses parâmetros apresente algumas diferenças.

Os estudos sobre a biologia dos triatomíneos possibilitam conhecer parâmetros que podem contribuir para os trabalhos de controle e manutenção de colônias em laboratório com os mais variados fins. Embora seja possível reproduzir as condições do habitat natural no laboratório, devem ser procuradas metodologias mais adequadas que interfiram menos nos resultados. Perlowagora-Szumlewicz (1976) demonstra a influência da geração utilizada e Tolezano et al. (1984 b) e Costa e Jurberg (1990) os efeitos do estresse causado pela manipulação dos insetos.

\section{REFERÊNCIAS}

Brasileiro VLF, Perondini ALP 1974. Biologia do T. brasiliensis (Hemiptera, Reduviidae, Triatominae). I. Tempo de sucção e repleção de ninfas de $4^{\circ}$ e $5^{\circ}$ estádios. Rev bras Ent 18: 43-50.

Cirano R, Zeledón R 1964. Observaciones sobre capacidad alimenticia y respiración de Triatoma infestans y Rhodnius prolixus (Hemiptera, Reduviidae). Rev Biol Trop 12: 271-285.

Corrêa RR, Espínola HN 1964. Descrição de Triatoma pseudomaculata, nova espécie de triatomíneo de Sobral, Ceará (Hemiptera: Reduviidae). Arq Higiene Saúde Públ São Paulo 29: 115-127.

Costa JM, Jurberg J 1990. Estudos bionômicos de Cavernicola lenti Barrett \& Arias, 1985 (Hemiptera, Reduviidae, Triatominae). Mem Inst Oswaldo Cruz 85: 357-366.

De Isola ED, Sanchez D, Katzin V 1980. Triatoma infestans: influencia de la alimentacion artificial sobre su ciclo de vida. Medicina 40 (Supl. 1): 207-212.

Dias JCP 1991. O controle da doença de Chagas no Brasil. Rev Soc Brasil Med Trop 24 (Supl. II): 201-202.

Erichson WF 1848. Insecten, p. 553-617. In Schomburgk, R, Reisen in British-Guiana, part 3. Versuch einer Fauna und Flora.

Forattini OP, Barata JMS, Santos JLF, Silveira AC 1981. Hábitos alimentares, infecção natural e distribuição de triatomíneos domiciliados na região nordeste do Brasil. Rev Saúde Púb S Paulo 15: 113-164.

Gonçalves TCM, Victório VMN, Jurberg J, Cunha V 1988. Biologia do Triatoma vitticeps (Stal, 1859) em condições de laboratório (Hemiptera: Reduviidae: Triatominae). I. Ciclo evolutivo. Mem Inst Oswaldo Cruz 83: 519-523.

Heitzmann-Fontenelle T 1972. Bionomia de Triatoma pseudomaculata Corrêa \& Espínola, 1964, em laboratório. Mem Inst Butantan 36: 251-262.

Heitzmann-Fontenelle T 1976. Bionomia comparativa de Triatomíneos. I - Panstrongylus megistus (Burm., 1835) (Hemiptera, Reduviidae). Studia Ent 19: 201210.

Juarez E 1970a. Observações sobre o ciclo evolutivo do Triatoma arthurneivai, em condições de laboratório (Hemiptera, Reduviidae). Rev Saúde Púb São Paulo 4: 13-18.

Juarez E 1970b. Comportamento do Triatoma infestans sob várias condições de laboratório. Rev Saúde Púb São Paulo 4: 147-166.

Krinsky WL 1985. Feeding, molting, and egg production in Rhodnius prolixus (Hemiptera: Reduviidae) fed repeatedly on the same swiss mouse hosts. J Med Entomol 22: 670-674.

Lent H, Valderrama A 1977. Observações sobre o ciclo evolutivo de Rhodnius prolixus Stal, 1859, Rhodnius pictipes Stal, 1872 e Rhodnius neivai Lent, 1953. Rev Bras Biol 37: 325-344.

Lucena DT 1959. Ecologia dos triatomíneos no Brasil. Rev brasil Malariol D trop 11: 577-635.

Maddrell SHP 1964. Excretion in the blood-sucking bug, Rhodnius prolixus Stal. II. The normal course of diuresis and the effect of temperature. J Exp Biol 41: 163-176.

Perlowagora-Szumlewicz A 1976. Laboratory colonies of Triatominae, biology and population dynamics. Scien Publ PAHO 318: 63-82.

Perlowagora-Szumlewicz A, Müller CA 1982. Studies in search of a suitable experimental insect model for xenodiagnosis of hosts with Chagas'disease. 1 Comparative xenodiagnosis with nine triatomine species of animals with acute infections by Trypanosoma cruzi. Mem Inst Oswaldo Cruz 77: 37-53.

Perlowagora-Szumlewicz A, Müller CA, Moreira CJC 1988. Studies in search of a suitable experimental insect model for xenodiagnosis of hosts with Chagas' disease. 3 - On the interaction of vectors species and parasite strain in the reaction of bugs to infection by Trypanosoma cruzi. Rev Saúde Púb S Paulo 22: 390-400.

Piesman J, Sherlock IA 1983. Factors controlling the volume produced by triatomine vectors of Chagas' disease. Acta Tropica 40: 351-358.

Silveira AC, Feitosa VR, Borges R 1984. Distribuição de triatomíneos capturados no ambiente domiciliar, no período 1975/83, Brasil. Rev brasil Malariol D trop 36: 15-312.

Tolezano JE, Chieffi PP, Araújo MFL de, Valentim AM, Ribeiro SS 1984a. Variáveis relacionadas ao desenvolvimento de Triatoma infestans Klug, 1834 em condições de laboratório. 1 - Relação entre 
repasto sanguíneo e desenvolvimento. Rev Inst Adolpho Lutz 44: 73-79.

Tolezano JE, Araújo MFL de, Chieffi PP, Valentim AM, Ribeiro, SS 1984b. Variáveis relacionadas ao desenvolvimento de Triatoma infestans Klug, 1834 em condições de laboratório. 2 - Efeitos da manipulação na sobrevivência e na fertilidade. Rev Inst Adolpho Lutz 44: 81-86.

Torres M 1915. Alguns fatos que interessam à epidemiolojia da molestia de Chagas. Mem Inst Oswaldo Cruz 7: 120.

Wood SF 1951. Importance of feeding and defecation times of insect vectors in transmission of Chagas' disease. J Econ Entomol 44: 52-54.

Zárate LG, López GM, Ozuna MC, Santiago GG, Zárate RJ 1984. The biology and behavior of Triatoma barberi (Hemiptera: Reduviidae) in Mexico. IV Feeding and defecation patterns. J Med Entomol 21: 548-560.

Zeledón R, Guardia VM, Zúñiga A, Swatzwelder JC 1970. Biology and ethology of Triatoma dimidiata (Latreille, 1811). I - Life cycle, amount of blood ingested, resistance to starvation, and size of adults. J Med Entomol 7: 313-319. 